\title{
Study on the Incidence of Stroke-Related Sarcopenia in Hospitalized Male Patients
}

\author{
Guang Liu' ${ }^{1}$ Chongwen Zheng², Donghao Zhou ${ }^{3}$ \\ ${ }^{1}$ Institute of Integrated Chinese and Western Medicine, Basic Medical College of Qingdao University, Qingdao, China \\ ${ }^{2}$ The Second People's Hospital of Fujian province, Xiamen, China \\ ${ }^{3}$ Department of Endocrinology and Metabolism, Linyi People's Hospital, Linyi, China \\ Email: zdh759@126.com
}

How to cite this paper: Liu, G., Zheng, C.W. and Zhou, D.H. (2020) Study on the Incidence of Stroke-Related Sarcopenia in Hospitalized Male Patients. Open Access Library Journal, 7: e7009.

https://doi.org/10.4236/oalib.1107009

Received: November 17, 2020

Accepted: December 28, 2020

Published: December 31, 2020

Copyright $\odot 2020$ by author(s) and Open Access Library Inc.

This work is licensed under the Creative Commons Attribution International License (CC BY 4.0).

http://creativecommons.org/licenses/by/4.0/

\section{(c) (i) Open Access}

\begin{abstract}
Objective To observe and analyze the incidence of sarcopenia in male patients with stroke-related sarcopenia. Methods From June 2018 to June 2019, the muscle mass of 45 patients who had recovered from stroke, who underwent DXA testing at Linyi People's Hospital during the period from June 2018 to June 2019. During the same period, 50 healthy subjects served as the control group to explore the muscles of stroke patients. Results The incidence of stroke-related sarcopenia was higher. The SMI, grip strength, upper and lower limb muscle mass, and calf circumference were significantly lower than those of patients with normal skeletal muscle mass and the control group $(P<0.05)$. Conclusion Stroke patients have a higher incidence of sarcopenia, and the occurrence of sarcopenia should be prevented during the recovery process.
\end{abstract}

\section{Subject Areas \\ Cardiology, Geriatrics}

\section{Keywords}

Sarcopenia, Stroke, Incidence

\section{Introduction}

The concept of stroke-related sarcopenia has been proposed in recent years. The disease is considered to be different from age-related sarcopenia. Studies have found that muscle structural changes begin to appear within a few hours after stroke, and then muscle mass decreases rapidly [1]. Park et al. [2] conducted a large-scale survey of 3009 men and 4199 women and found a strong positive 
correlation between stroke and sarcopenia. This study intends to investigate the incidence of stroke-related sarcopenia in hospitalized stroke patients recovering from stroke.

\section{Objects and Methods}

\subsection{Research Objects}

45 stroke male patients hospitalized in Linyi People's Hospital from June 2018 to June 2019 were set as the observation group, and 50 healthy men with physical examination at the same time period were selected as the control group. This study has been reviewed by the ethics committee of our hospital.

Inclusion criteria: 1) Patients who meet the diagnosis of stroke; 2) Patients who meet the diagnosis of sarcopenia.

Exclusion criteria: 1) Patients with other diseases; 2) Patients with long-term use of hormones and other drugs that cause sarcopenia.

Sarcopenia refers to the 2019 diagnostic criteria of the Asian Sarcopenia Working Group [3]: Dual-energy X-ray examination of male skeletal muscle mass in$\operatorname{dex}(\mathrm{SMI})<7.0 \mathrm{~kg} / \mathrm{m}^{2}$, female SMI $<5.4 \mathrm{~kg} / \mathrm{m}^{2}$; male grip strength $<28 \mathrm{~kg}$, female Grip strength $<18 \mathrm{~kg}$; calf circumference: male $<34 \mathrm{~cm}$, female $<33 \mathrm{~cm}$.

\subsection{Measurement Method}

Use the Lunar dual-energy X-ray bone densitometer (DXA) from GE Company to measure the body muscle mass. After removing the metal objects worn by all patients, they lie supine on the measuring bed at room temperature and rest, and measure the muscle mass of the whole body according to the DXA procedure. A grip device (EH101, Xiangshan, China) was used to test the grip strength. The subject was in a standing position and the elbow extension was tested; if the subject could not stand independently, a sitting position was used for the test. Use the dominant hand or both hands to perform the test with the maximum force, at least 2 times, and select the maximum value.

\subsection{Statistical Processing}

Using SPSS22.0 statistical software, t-test or rank-sum test was used for comparison of measurement data between groups, and chi-square test was used for count data.

\section{Results}

A total of sarcopenia and pre-sarcopenia were detected in the observation group, and a total of sarcopenia and pre-sarcopenia were detected in the control group. The incidence of the observation group was significantly higher than that of the control group (see Table 1).

The SMI, grip strength, skeletal muscle mass of the upper and lower limbs, and calf circumference of the observation group of sarcopenia patients were significantly lower than those of non sarcopenia patients (see Table 2). 
Table 1. The incidence of sarcopenia in the two groups.

\begin{tabular}{ccccc}
\hline & Cases & sarcopenia & pre-sarcopenia & non sarcopenia \\
\hline Observation group & 45 & 15 & 11 & 19 \\
Control group & 50 & 5 & 7 & 38 \\
$\chi^{2}$ & & 7.76 & 1.68 & 11.26 \\
$P$ & & 0.005 & 0.20 & 0.001 \\
\hline
\end{tabular}

Table 2. Comparison of basic data of the two groups of subjects $(\bar{x} \pm s)$.

\begin{tabular}{|c|c|c|c|c|c|c|}
\hline & \multicolumn{3}{|c|}{ Observation group } & \multicolumn{3}{|c|}{ Control group } \\
\hline & non sarcopenia & pre-sarcopenia & sarcopenia & non sarcopenia & pre-sarcopenia & sarcopenia \\
\hline $\operatorname{SMI}\left(\mathrm{kg} / \mathrm{m}^{2}\right)$ & $8.04 \pm 0.62$ & $6.42 \pm 0.68^{\mathrm{a}}$ & $6.50 \pm 0.36^{\mathrm{a}}$ & $8.01 \pm 0.62$ & $6.62 \pm 0.65^{\mathrm{a}}$ & $6.60 \pm 0.35^{\mathrm{a}}$ \\
\hline Upper limb muscle mass (kg) & $6.60 \pm 0.98$ & $4.54 \pm 0.83^{\mathrm{a}}$ & $5.01 \pm 0.51^{\mathrm{a}}$ & $6.37 \pm 0.98$ & $4.86 \pm 0.70^{\mathrm{a}}$ & $5.30 \pm 0.38^{\mathrm{a}}$ \\
\hline Lower limb muscle mass (kg) & $17.34 \pm 2.01$ & $12.84 \pm 2.19^{\mathrm{a}}$ & $14.03 \pm 1.33^{\mathrm{a}}$ & $16.98 \pm 1.88$ & $14.01 \pm 1.88^{\mathrm{a}}$ & $14.36 \pm 1.08^{\mathrm{a}}$ \\
\hline BMI $\left(\mathrm{kg} / \mathrm{m}^{2}\right)$ & $27.40 \pm 2.04$ & $25.25 \pm 2.30^{\mathrm{a}}$ & $23.57 \pm 2.51^{\mathrm{a}}$ & $26.33 \pm 2.98$ & $25.63 \pm 2.45$ & $22.69 \pm 1.89^{\mathrm{a}}$ \\
\hline Grip strength (kg) & $36.06 \pm 10.64$ & $36.46 \pm 5.48$ & $23.62 \pm 9.46^{\mathrm{ab}}$ & $36.97 \pm 10.47$ & $36.76 \pm 7.29$ & $25.44 \pm 8.56^{\mathrm{ab}}$ \\
\hline Calf circumference $(\mathrm{cm})$ & $37.37 \pm 2.99$ & $33.91 \pm 3.07^{\mathrm{a}}$ & $33.73 \pm 2.67^{\mathrm{a}}$ & $36.53 \pm 2.79$ & $34.36 \pm 3.46$ & $34.00 \pm 2.45$ \\
\hline
\end{tabular}

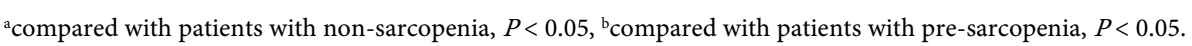

\section{Discussion}

This study shows that the prevalence of sarcopenia is higher among stroke rehabilitation patients, which is significantly higher than that of the general population. Ryan et al. [4] studied 190 patients 6 months after the onset of stroke and showed that the prevalence of sarcopenia in stroke patients was between $14 \%$ and $18 \%$. Jyunya et al. [5] conducted a study on 117 chronic stroke survivors 65 years of age or older, and detected a total of 60 sarcopenia patients, and found that sarcopenia patients are mainly knee extension weakness and ankle weakness. The assessment and intervention of skeletal muscle in patients with stroke sarcopenia should focus on the knee and ankle joints. Su et al. [6] found that the combined prevalence of stroke-related sarcopenia was $42 \%$. In this study, the incidence of sarcopenia was $33.33 \%$, and the incidence of pre-sarcopenia was $24.44 \%$, which was higher than healthy people.

The study found that the number of motor units in patients with cerebral infarction began to decrease 4 hours after cerebral infarction, and the number of motor units in muscle tissue decreased significantly within 30 hours after cerebral infarction. The reason may be due to the synapses of the spinal cord alpha motor neurons that innervate the muscle was suppressed [7]. Deyne et al. [8] found that in contrast to the transition of the fiber type in elderly patients with sarcopenia from fast muscle contraction fibers to slow muscle contraction fibers, in stroke patients, slow muscle contraction fibers were observed to be fast muscle contraction dominated by anaerobic metabolism. The reverse transition of fiber MHC type II subtype, and the proportion of fast muscle contraction fiber 
MHC type II is inversely proportional to the severity of gait defect. Matsushita et al. [9] showed that stroke-related sarcopenia seems to be a predictor of how male patients will engage in activities of daily living after recovery.

\section{Conclusion}

In summary, the incidence of stroke-related sarcopenia is relatively high, and it is not significantly affected by age, and has an important impact on the prognosis of patients. Therefore, it is necessary to pay special attention to the screening of sarcopenia for stroke rehabilitation patients in clinic.

\section{Conflicts of Interest}

The authors declare no conflicts of interest regarding the publication of this paper.

\section{References}

[1] Scherbakov, N., Sandek, A., Doehner, W., et al. (2015) Stroke-Related Sarcopenia: Specific Characteristics. Journal of the American Medical Directors Association, 16, 272-276. https://doi.org/10.1016/j.jamda.2014.12.007

[2] Park, S., Ham, J.O. and Lee, B.K. (2014) A Positive Association between Stroke Risk and Sarcopenia in Men Aged $\geq 50$ Years, But Not Women: Results from the Korean National Health and Nutrition Examination Survey 2008-2010. The Journal of $\mathrm{Nu}$ trition, Health \& Aging, 18, 806-812. https://doi.org/10.1007/s12603-014-0553-x

[3] Chen, L.K., Woo, J., Assantachai, P., et al. (2020) Asian Working Group for Sarcopenia: 2019 Consensus Update on Sarcopenia Diagnosis and Treatment. Journal of the American Medical Directors Association, 21, 300-307. https://doi.org/10.1016/j.jamda.2019.12.012

[4] Ryan, A.S., Ivey, F.M., Serra, M.C., et al. (2017) Sarcopenia and Physical Function in Middle-Aged and Older Stroke Survivors. Archives of Physical Medicine and Rehabilitation, 98, 495-499. https://doi.org/10.1016/j.apmr.2016.07.015

[5] Jyunya, Y. (2020) Stroke Sarcopenia Patients Cause Weakness and Atrophy in the Knee and Ankle Joints. Current Developments in Nutrition, 4, 214.

https://doi.org/10.1093/cdn/nzaa043 065

[6] Su, Y., Yuki, M. and Otsuki, M. (2020) Prevalence of Stroke-Related Sarcopenia: A Systematic Review and Meta-Analysis. Journal of Stroke and Cerebrovascular Diseases, 29, Article ID: 105092.

https://doi.org/10.1016/j.jstrokecerebrovasdis.2020.105092

[7] Arasaki, K., Igarashi, O., Ichikawa, Y., et al. (2006) Reduction in the Motor Unit Number Estimate (MUNE) after Cerebral Infarction. Journal of the Neurological Sciences, 250, 27-32. https://doi.org/10.1016/j.jns.2006.06.024

[8] De Deyne, P.G., Hafer-Macko, C.E., Ivey, F.M., et al. (2004) Muscle Molecular Phenotype after Stroke Is Associated with Gait Speed. Muscle \& Nerve, 30, 209-215. https://doi.org/10.1002/mus.20085

[9] Matsushita, T., Nishioka, S., Taguchi, S., et al. (2019) Sarcopenia as a Predictor of Activities of Daily Living Capability in Stroke Patients Undergoing Rehabilitation. Epidemiology, Clinical Practice and Health, 19, 1124-1128. https://doi.org/10.1111/ggi.13780 